\title{
Characterizing dynamic behaviors of three-particle paramagnetic microswimmer near a solid surface
}

\author{
Qianqian Wang ${ }^{1}$, Lidong Yang ${ }^{1}$, Jiangfan Yu ${ }^{1}$ and Li Zhang $1,2,3^{*}$
}

\begin{abstract}
Particle-based magnetically actuated microswimmers have the potential to act as microrobotic tools for biomedical applications. In this paper, we report the dynamic behaviors of a three-particle paramagnetic microswimmer. Actuated by a rotating magnetic field with different frequencies, the microswimmer exhibits simple rotation and propulsion. When the input frequency is below $8 \mathrm{~Hz}$, it exhibits simple rotation on the substrate, whereas it shows propulsion with varied poses when subjected to a frequency between 8 and $15 \mathrm{~Hz}$. Furthermore, a solid surface that enhances swimming velocity was observed as the microswimmer is actuated near a solid surface. Our simulation results testify that the surface-enhanced swimming near a solid surface is because of the induced pressure difference in the surrounding fluid of the microagent.
\end{abstract}

Keywords: Swimming microrobot, Magnetic actuation, Boundary effect, Low Reynolds number, Dynamic behavior

\section{Introduction}

Microswimmers remotely actuated by magnetic fields have been considered as promising microrobotic tools because of their great potential in biomedical applications [1], such as targeted therapy [2], drug delivery [3, 4] and minimally invasive surgery [5]. Various designs of microswimmers combined with diverse magnetic actuation strategies have been proposed [6-10]. Among them, inspired by $E$. coil bacterial, helical microswimmer has drawn attention of many researchers. For propulsion of helical microswimmers, rotating magnetic fields are widely used for the generation of corkscrew motion at low Reynolds number. It was reported that, actuated by a rotating magnetic field, the "artificial bacterial flagella" (ABF) perform versatile swimming behaviors and can act as effective tools for cargo transport and micromanipulation tasks [11-15]. These ABF were fabricated using self-scrolling technique [11], 3-D direct laser writing [14], glancing angle deposition technique [16, 17], DNA-based

\footnotetext{
*Correspondence: lizhang@mae.cuhk.edu.hk

1 Department of Mechanical and Automation Engineering, The Chinese

University of Hong Kong, Shatin, Hong Kong SAR, China

Full list of author information is available at the end of the article
}

flagellar bundles [18], and so on. The dynamics of such helical swimmers have been studied systematically below, near and higher than the step-out frequency. For instance, to perform corkscrew motion with continuous rotation, usually a magnetic helical swimmer should be actuated with an input frequency that is below its stepout frequency, whereas the actuation with a frequency that is higher than the step-out frequency will lead to a so-called "jerky motion" $[19,20]$, i.e., the combination of a rotation with stops and backward motions [21], which results in a decrease in its translational velocity [12, 2224]. Interestingly, Ghosh et al. [24] reported that a helical microswimmer could exhibit bistable behaviors under an external field near the step-out frequency, showing random switch between two configurations, i.e., propulsion or tumbling motion.

Unlike the propulsion of tiny structures with chirality in low Reynolds number regime, it has been demonstrated recently that randomly shaped microswimmers can also be actuated effectively using a rotating magnetic field $[25,26]$. These microswimmers are obtained using iron oxide nanoparticle aggregations with varied shapes based on hydrothermal carbonization. Alternatively, Cheang et al. [27] reported that achiral three-particle 
microswimmers exhibit controlled swimming motion under a rotating magnetic field. These microswimmers consist of three polystyrene microparticles embedded with paramagnetic or ferromagnetic nanoparticles, and varied swimming behaviors are triggered because of their different magnetic properties, despite the geometrical similarity.

It is notable that recent studies of three-particle microswimmers focus on swimming behaviors in fluid with negligible boundary effects [27-29]; however, their swimming behaviors near a solid surface can be significantly affected due to the boundary effect. Previously, the boundary effects were reported on both natural swimming organisms and artificial swimmers. The influence of solid boundaries has been observed and analyzed for E. coil bacteria [30, 31], and spermatozoa self-organized into dynamic vortices resembling quantized rotating waves on a planar surface [32]. A solid surface affects swimming direction of $\mathrm{ABF}$, resulting in drifting behaviors [14], and wobbling motion of the ABF enhances the sidewise drift due to wall effects [33]. Simulation results indicate that microswimmer exhibits enhanced mobility when swimming between inclined rigid boundaries [34], and a surface can deform the induced streamlines of a rotating microagent [35].

Here, we report the dynamic behaviors of a paramagnetic three-particle microswimmer, which is actuated near a solid surface using a rotating magnetic field. With rotation axis of the magnetic field perpendicular to the horizontal surface, the microswimmer exhibits simple rotation when the input frequency is below $8 \mathrm{~Hz}$, whereas it shows propulsion when subjected to a frequency between 8 and $15 \mathrm{~Hz}$ (Fig. 1). Furthermore, enhanced swimming velocity can be achieved if the microswimmer exhibits propulsion near the surface, because of the induced pressure difference in the surrounding fluid of the

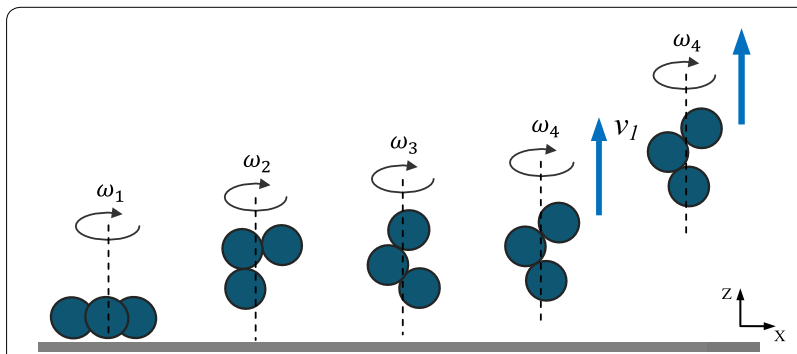

Fig. 1 Schematic of the dynamic behaviors of a three-particle microswimmer under a rotating magnetic field. Black dashed line and arrows refer to the rotation axis and direction with angular velocity $\omega_{1}<\omega_{2}<\omega_{3}<\omega_{4}$, blue arrows refer to velocity with $v_{1}>v_{2}$. The microswimmer exhibits simple rotation $\left(\omega_{1}\right)$ and propulsion under different input frequencies $\left(\omega_{2}, \omega_{3}, \omega_{4}\right)$. Gray rectangle refers to a solid surface microswimmer. While with the rotation axis of the field parallel to the surface, the microswimmer exhibits lowfrequency tumbling $(1-3 \mathrm{~Hz})$ and wobbling $(3-15 \mathrm{~Hz})$. The main contributions of this work include the following two aspects. First, a mathematical model is proposed for the analysis of dynamic poses under different input frequencies. Second, simulation results show that the induced pressure near a surface can enhance swimming velocity of a three-particle microswimmer, which are validated by experimental results.

The remaining parts of this paper are structured as follows. Mathematical modeling and simulations of the microswimmer are presented in Methods section. Then, in section Results and discussion, we discuss the dynamic behaviors of this microswimmer, and the experimental results are analyzed as well. Finally, Conclusions are given in the last section.

\section{Methods}

\section{Mathematical modeling}

The three-particle microswimmer is treated as a rigid structure with two perpendicular planes of symmetry, forms an achiral structure (Fig. 2a). It is placed on a solid surface and actuated by a rotating magnetic field (Fig. 2b).

\section{Motion at low Reynolds numbers}

The hydrodynamics of the microswimmer in low Reynolds number regime can be described by the Stokes equations:

$$
\begin{aligned}
& \eta \nabla^{2} \mathbf{u}-\nabla p=0 \\
& \nabla \cdot u=0
\end{aligned}
$$

where $p$ is pressure and $u$ is moving velocity of the fluid. The relationship of external force $\boldsymbol{F}$ together with torque $\boldsymbol{\tau}$ and translational velocity $\boldsymbol{V}$ together with angular velocity $\omega$ is described as [36]:

$$
\left[\begin{array}{c}
V \\
\omega
\end{array}\right]=\left[\begin{array}{cc}
K & C_{o} \\
C_{o}^{T} & \Omega_{o}
\end{array}\right]\left[\begin{array}{c}
F \\
\tau
\end{array}\right]
$$

where $\boldsymbol{K}$ is the translation tensor and $\boldsymbol{\Omega}_{\boldsymbol{o}}$ is the rotation tensor. $\boldsymbol{C}_{\boldsymbol{o}}$ is the coupling tensor, representing coupling of translational and rotational motions of a microagent. For the microagent in Fig. 2a, the matrices $\boldsymbol{K}, \boldsymbol{\Omega}_{\boldsymbol{o}}$ and $\boldsymbol{C}_{\boldsymbol{o}}$ are given by

$$
\begin{array}{ll}
\mathbf{K} & =\left[\begin{array}{lll}
K_{1} & 0 & 0 \\
0 & K_{2} & 0 \\
0 & 0 & K_{3}
\end{array}\right], \quad \Omega_{\mathbf{o}}=\left[\begin{array}{ccc}
\Omega_{1} & 0 & 0 \\
0 & \Omega_{2} & 0 \\
0 & 0 & \Omega_{3}
\end{array}\right], \\
\mathbf{C}_{\mathbf{o}} & =\left[\begin{array}{lll}
0 & 0 & 0 \\
0 & 0 & C_{23} \\
0 & C_{32} & 0
\end{array}\right]
\end{array}
$$



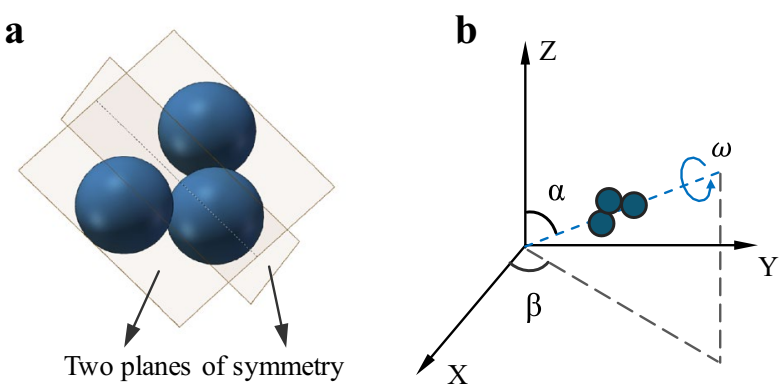

Fig. 2 Structure of the microswimmer and the applied magnetic field. a Microswimmer is treated as a rigid structure with two mutually perpendicular planes of symmetry. $\mathbf{b}$ Schematic of the rotating magnetic field with constant flux density. Blue dashed line and arrow refer to the normal line and rotation direction of the magnetic field, respectively. Pitch angle $\alpha$ is between the normal line and Z-axis, and yaw angle $\beta$ is between $X$-axis and projection of the normal line in the $X Y$-plane

\section{Magnetic actuation}

The magnetic force and torque exerted on the microswimmer are given by:

$$
\begin{aligned}
& \boldsymbol{F}=(\boldsymbol{m} \cdot \nabla) \boldsymbol{B} \\
& \boldsymbol{\tau}=\boldsymbol{m} \times \boldsymbol{B}
\end{aligned}
$$

where $\boldsymbol{m}$ is the induced magnetic dipole moment and $\boldsymbol{B}$ is the flux density of the magnetic field. Here we have $\boldsymbol{F}=0$ because the applied magnetic field has uniform flux density. The angular and translational velocity of the microswimmer due to induced magnetic torque is denoted by

$$
\begin{aligned}
& \omega=\boldsymbol{\Omega}_{\boldsymbol{o}}(\boldsymbol{m} \times B) \\
& V=C_{o}(m \times B)
\end{aligned}
$$

Two equations above indicate that if the coupling tensor $\boldsymbol{C}_{\boldsymbol{o}}$ is nonzero, a rotating microswimmer can exhibit translational velocity.

Next, we show the two torques (i.e., drag torque and magnetic torque) counterbalanced with each other. When the pitch angle $\alpha$ is $0, \boldsymbol{m}$ and $\boldsymbol{B}$ are both in a plane perpendicular to the $Z$-axis (Fig. 3 ), making the angular velocity $\boldsymbol{\omega}=\left[\begin{array}{lll}0 & 0 & \omega_{z}\end{array}\right]^{T}$ and magnetic torque $\boldsymbol{\tau}_{\boldsymbol{m}}=\left[\begin{array}{lll}0 & 0 & \tau_{m z}\end{array}\right]^{T}$. The induced magnetic torque can be treated as the torque exerted on a chain that consists of three spherical particles [37], as expressed by

$$
\tau_{m z}=\frac{3}{4} \pi a^{3} \mu_{0} \chi^{2} B^{2} \sin (2 \theta)
$$

where $a$ is radius of the particle, $\mu_{0}$ is the vacuum permeability, $\chi$ is the particle susceptibility and $\theta$ is the phase lag between external field and induced dipole moment. If the microswimmer is actuated with steady rotation, the phase lag must satisfy the condition $\sin (2 \theta)<1$ [37]. The drag torque $\boldsymbol{\tau}_{\boldsymbol{r}}$ due to hydrodynamic interaction can be

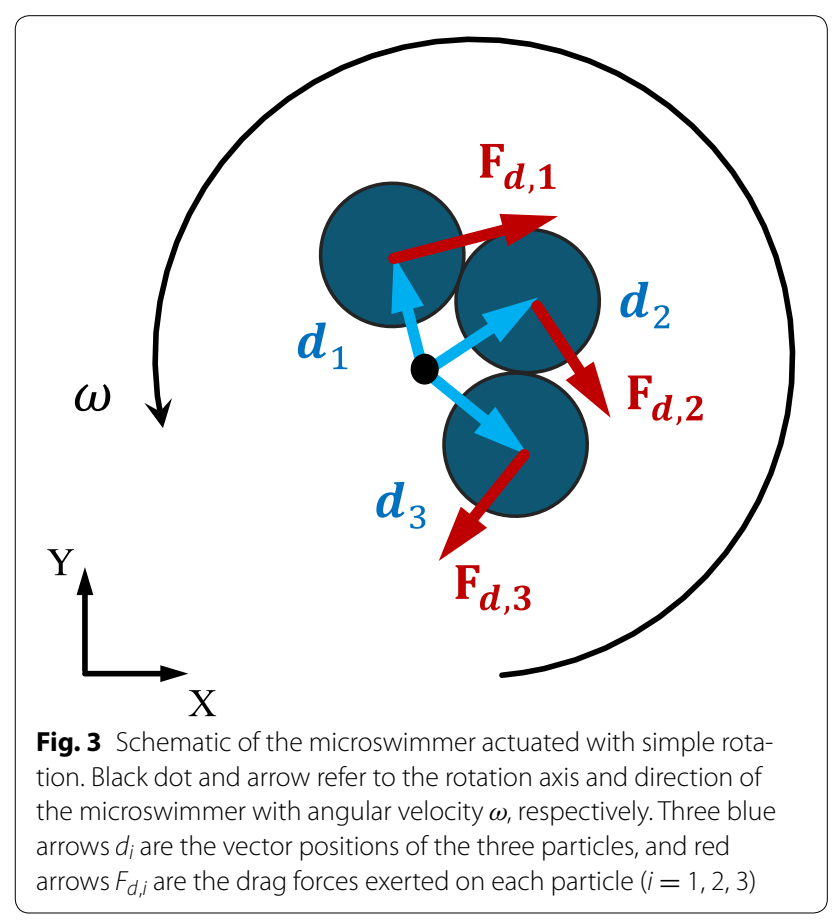

obtained by combining torque from each particle individually [38]. Similarly, we have $\boldsymbol{\tau}_{\boldsymbol{r}}=\left[\begin{array}{lll}0 & 0 & \tau_{r z}\end{array}\right]^{T}$. For each particle, the drag torque is given by

$$
\begin{aligned}
& \boldsymbol{\tau}_{r z, i}=\boldsymbol{d}_{\boldsymbol{i}} \times \boldsymbol{F}_{\boldsymbol{d}, \boldsymbol{i}} \\
& \boldsymbol{F}_{\boldsymbol{d}, \boldsymbol{i}}=D_{d} \boldsymbol{V}_{\boldsymbol{i}}=D_{d}\left(\boldsymbol{\omega}_{z} \times \boldsymbol{d}_{\boldsymbol{i}}\right)
\end{aligned}
$$

where $\boldsymbol{d}_{\boldsymbol{i}}$ and $\boldsymbol{F}_{\boldsymbol{d}, \boldsymbol{i}}$ are vector position and drag force of the $i$ - th microparticle, and $D_{d}$ is the drag force coefficient, respectively. For spherical microparticles without any effects from boundary $D_{d}=-6 \pi \eta a$. The total drag torque is given by 


$$
\boldsymbol{\tau}_{\boldsymbol{r} z}=\sum_{n=1}^{3} \boldsymbol{d}_{\boldsymbol{i}} \times \boldsymbol{F}_{\boldsymbol{d}, \boldsymbol{i}}=D_{d} \boldsymbol{\omega}_{z} \sum_{n=1}^{3} d_{i}^{2}
$$

Eq.12 shows that larger $\sum_{i=1}^{3} d_{i}^{2}$ leads to larger drag torque under the same input frequency.

\section{Pose change frequency}

The microswimmer undergoes constant magnetic torque due to uniform magnetic flux density. However, the drag torque is in dependence on the input frequency of magnetic field and rotation pose of the microswimmer. Next, from the torque-balance perspective, we show how swimming behaviors of our microswimmer varied by increasing the input frequency. The phase lag for a given input frequency and magnetic flux density is [37]

$$
\sin (2 \theta)=\frac{96 \eta \omega}{\mu_{0} \chi^{2} B^{2} \ln \left(\frac{3}{2}\right)}
$$

In order to balance the two torques, term $\sum_{i=1}^{3} d_{i}^{2}$ in Eq. 12 must change its value corresponding to different input frequencies, which results in different rotation poses of the microswimmer. However, the adjustable range of this term has limitation. Let us consider two cases of the microswimmer under actuation, i.e., simple rotation and propulsion. We simplify the microswimmer as an isosceles triangle with two sides of identical length $L$ and the included angle $\gamma$. Since we only consider microswimmer with two perpendicular planes of symmetry, $\gamma$ in our analysis is set to be $\pi / 3<\gamma<\pi$.

First, we assume that the microswimmer is actuated with simple rotation as shown in Fig. 4a. From the top view, the rotation axis is a dot with coordinate $\left(x_{r}, y_{r}\right)$. From geometrical perspective, we have

$$
\sum_{n=1}^{3} d_{i}^{2}=\left(x_{i}-x_{r}\right)^{2}+\left(y_{i}-y_{r}\right)^{2}
$$

where $\left(x_{i}, y_{i}\right)$ is the coordinate of the $i-t h$ particle's center. The minimal value of $\sum_{i=1}^{3} d_{i}^{2}$ exists if the rotation axis passes through the centroid of the simplified isosceles triangle, given by

$$
\left(x_{c}, y_{c}\right)=\left(\frac{\sum_{n=1}^{3} x_{i}}{3}, \frac{\sum_{n=1}^{3} y_{i}}{3}\right)
$$

Substituting Eq. 15 into Eq. 14 yields

$$
\begin{aligned}
& \sum_{n=1}^{3} d_{i \min }^{2}=\frac{2}{3} L^{2}(2+\sin \gamma-\cos \gamma) \\
& \sum_{n=1}^{3} d_{i \min }^{2} \in\left(1.58 L^{2}, 2.28 L^{2}\right)
\end{aligned}
$$

Then, we assume the microswimmer is actuated with propulsion as shown in Fig. 4b. In this scenario, the minimal value exists if the rotation axis is parallel to the longest side of the triangle, which is the side respected to angle $\gamma$ since $\gamma>\pi / 3$. Calculation results show that the minimal value exists when the rotation axis passes through the point $p$, a point of trisection of the height with respect to the longest side. Similarly, we have $\mathbf{a}$

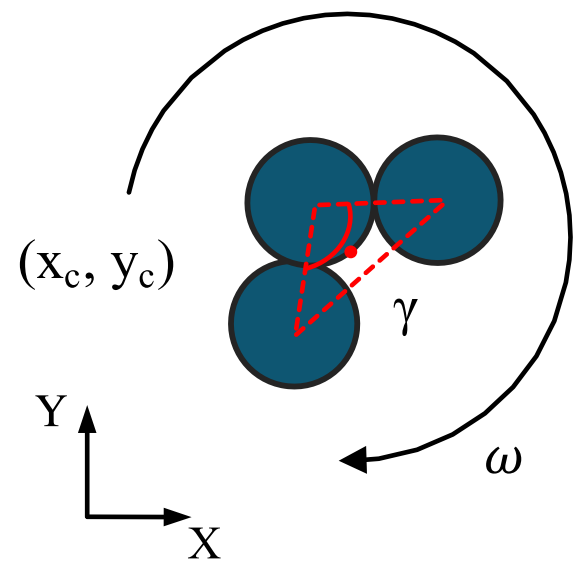

b

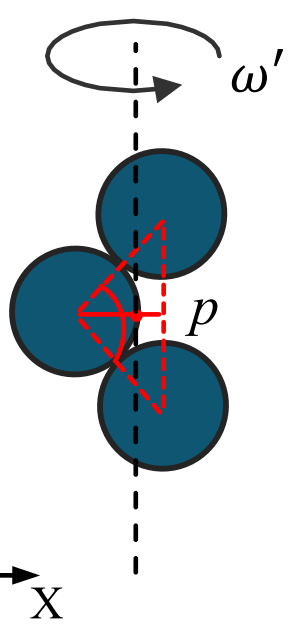

c

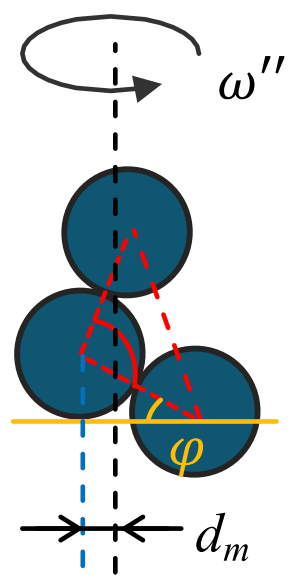

Fig. 4 a Schematic of the microswimmer actuated with simple rotation. Rotation axis of the microswimmer coincides with centroid of the simplified isosceles triangle (red dash lines). The red dot refers to the projection of the rotation axis as well as the centroid with coordinate $\left(x_{c}, y_{c}\right.$ ). b Rotation axis of the microswimmer coincides with the longest side of the simplified isosceles triangle. $\mathbf{c}$ Dynamic behaviors with pose angle $\varphi$ and distance $d_{m}$. Black arrows and dash lines refer to the angular velocity and rotation axis of the microswimmer, respectively. $\mathbf{b}, \mathbf{c}$ Have the same coordinate 


$$
\begin{aligned}
& \sum_{n=1}^{3} d_{i \min }^{2}=\frac{2}{3} L^{2} \cos ^{2} \frac{\gamma}{2} \\
& \sum_{n=1}^{3} d_{i \min }^{2} \in\left(0, \frac{1}{2} L^{2}\right)
\end{aligned}
$$

The analysis results above, in particular Eqs. 17 and 19, show that with the same input frequency, drag torque becomes smaller if the microswimmer exhibits propulsion rather than simple rotation. Finally, let us consider a specific case. We increase the input frequency continuously, at first, the microswimmer exhibits simple rotation, and then, it tends to change its actuation behaviors toward reducing the drag torque. The only feasible method is to reduce the distance (term $\sum_{i=1}^{3} d_{i}^{2}$ in Eq. 12) between each microparticle and the rotation axis. Therefore, the microswimmer has to change from simple rotation to propulsion when the input frequency is higher than a certain value $\omega_{c}$, and here we name $\omega_{c}$ as the pose-change frequency. When the angular velocity is high enough, the propulsion force of the microswimmer is larger than the combination of gravitational force and buoyancy, so that it will swim. A switch from simple rotation to propulsion can be realized by increasing the input frequencies with a value higher than $\omega_{c}$. For example in Fig. $1, \omega_{1}$ is below $\omega_{c}$ while $\omega_{2}, \omega_{3}, \omega_{4}$ are higher than $\omega_{c}$.

Besides the two specific scenarios shown in Fig. 4a, $\mathrm{b}$, other dynamic behaviors can be realized as well. As shown in Fig. 4c, we define the simplified triangular has an angle $\varphi$ with $X$-axis and the distance between the vertex and rotation axis is $d_{m}$. These two parameters are able to represent the propulsion pose with rotation axis. Here the $\sum_{i=1}^{3} d_{i}^{2}$ is calculated as

$$
\begin{aligned}
\sum_{i=1}^{3} d_{i}^{2}= & 3 d_{m}^{2}+L^{2}\left[\cos ^{2}(\varphi)+\cos ^{2}(\varphi-\gamma)\right] \\
& -2 L d_{m}[\cos (\varphi)+\cos (\varphi-\gamma)]
\end{aligned}
$$

For a given microswimmer $\gamma=\pi / 2$, if we define $d_{m}=\sigma L(0 \leq \sigma \leq 1)$ and Eq. 20 can be simplified as

$$
\sum_{i=1}^{3} d_{i}^{2}=L^{2}\left[3 \sigma^{2}+1-2 \sigma(\sin \varphi+\cos \varphi)\right]
$$

The range of $\varphi$ is set to be $0 \leq \varphi \leq \pi / 4$, while $\pi / 4 \leq \varphi \leq \pi / 2$ results in the same value of $\sum_{i=1}^{3} d_{i}^{2}$ because of the symmetry of the model. We use MATLAB to calculate the distribution of the value in Eq. 21, and the results are shown in Fig. 5. The maximum value exists when $\sigma=1$ and $\varphi=0$, corresponding to the pose with angular velocity $\omega_{2}$ in Fig. 1 . Interestingly, the minimum value is $\sum_{i=1}^{3} d_{i}^{2}=L^{2} / 3$ when $\sigma=0.47$ and $\varphi=\pi / 4$,

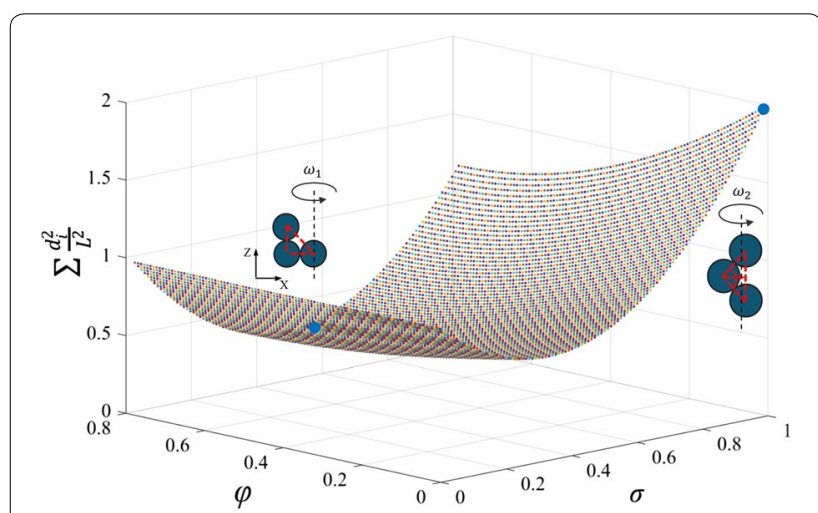

Fig. 5 Distribution of the value of $\sum_{i=1}^{3} d_{i}^{2}$. The minimum and maximum values are marked with the corresponding rotation poses. The two insets have the same coordinate

which also proves that minimal value exists when the rotation axis is parallel to the longest side of the simplified triangle model.

\section{Simulations}

To simulate and understand how a solid surface affects swimming behaviors, two finite element method (FEM) models are established using COMSOL Multiphysics (two insets in Fig. 6a, d) to investigate the induced fluid flows (Fig. 6a, b) and pressure (Fig. 6b, c, e, f) by the rotating microswimmer. The microswimmer is modeled as three spheres with a diameter of $4.5 \mu \mathrm{m}$ and an angle $\gamma=\pi / 2$, and set to be actuated in water at a frequency of $10 \mathrm{~Hz}$. A solid surface is modeled as a no-slip wall at the bottom. Simulations consist of two cases: Fig. $6 \mathrm{a}-\mathrm{c}$ are simulation results with microswimmer near $(0.75 \mu \mathrm{m})$ the surface, and Fig. $6 \mathrm{~d}-\mathrm{f}$ are results with it farther away $(20.75 \mu \mathrm{m})$ from the surface. After over ten full rotations, the induced pressure distribution and streamlines of the surrounding fluid are calculated. Figure $6 \mathrm{a}, \mathrm{b}$ show that the microswimmer induces a net flow of fluid along the direction of the rotation axis, similar to the propulsion of a helical flagellum [15, 39]. The fluid impacts on the substrate, resulting in enhanced pressure [40]. For the case of rotation near the surface, the induced pressure difference between the area near the top and bottom space of the microswimmer is observed in Fig. 6b, c. However, such difference becomes negligible when the microswimmer $20.75 \mu \mathrm{m}$ above the surface (Fig. 6e, f). The largest pressure difference around each particle is in the order of $10^{-2} \mathrm{~Pa}$ (Fig. 7). The affected area on the microswimmer is in the order of $10^{1} \mu \mathrm{m}^{2}$ and the net force along $Z$-axis works out in piconewton range due to the pressure difference. 


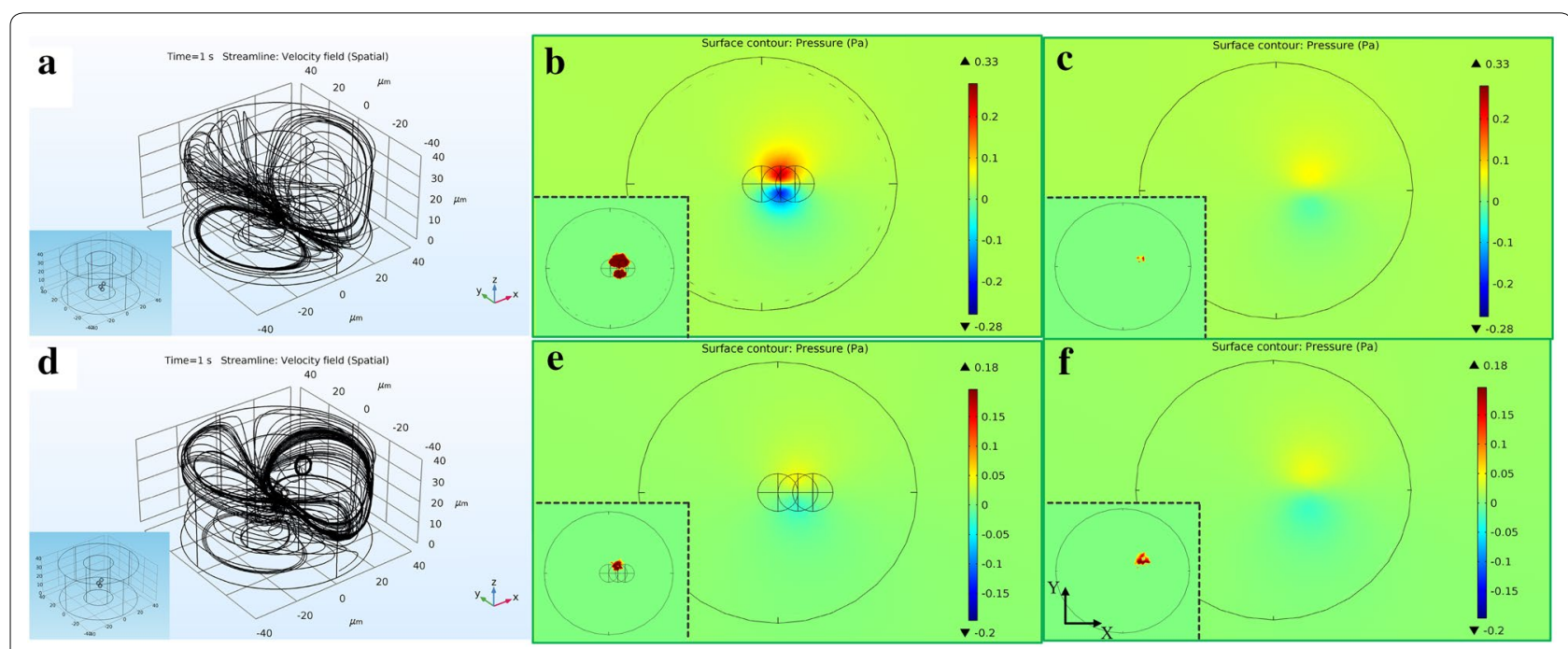

Fig. 6 Simulation of the microswimmer rotates above a no-slip wall. Rotation axis is defined as Z-axis; numbers represent dimensions in micrometer. The microswimmer with two different boundary conditions is modeled $\mathbf{a} 0.75 \mu \mathrm{m}$ and $\mathbf{d} 20.75 \mu \mathrm{m}$ above the no-slip wall, as shown in the insets of $\mathbf{a}$ and $\mathbf{d}$, respectively. $\mathbf{a}, \mathbf{b}$ The streamlines generated by the microswimmer. $\mathbf{b}, \mathbf{c}, \mathbf{e}, \mathbf{f}$ Pressure induced by rotation of the microswimmer at a frequency of $10 \mathrm{~Hz}$ in a plane $0.5 \mu \mathrm{m}$ below $\mathbf{b}$, e and above $\mathbf{c}, \mathbf{f}$ the microswimmer, $\mathbf{b}$, $\mathbf{c}$ are with the microswimmer $0.75 \mu \mathrm{m}$ above the no-slip wall, and $\mathbf{e}, \mathbf{f}$ are $20.75 \mu \mathrm{m}$ above the no-slip wall. The color legends in the main frame illustrate the magnitude of pressure (Pa). The red areas of insets in b, e are with pressure higher than $0.1 \mathrm{~Pa}$, and those in $\mathbf{e}, \mathbf{f}$ are higher than $0.04 \mathrm{~Pa}$. b, $\mathbf{c}, \mathbf{e}, \mathbf{f}$ with all insets have the same coordinate

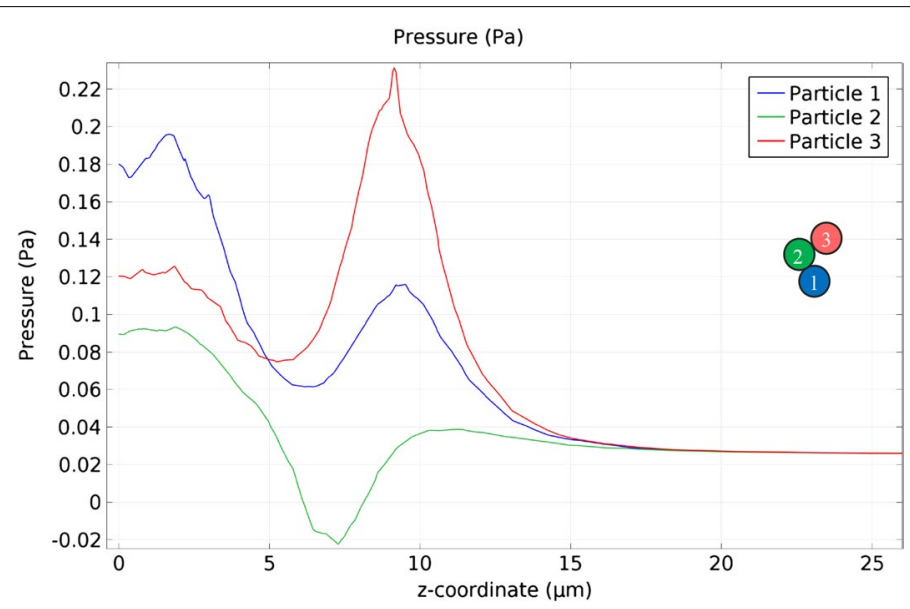

Fig. 7 Simulation results of the pressure distribution. Pressure distribution near the microswimmer. Three lines denotes the pressure near the three particles in blue, green and red, respectively

\section{The microswimmer and experimental setup}

In our experiments, the microswimmer was obtained by direct sediment of paramagnetic microparticles colloidal suspensions (Spherotech PMS-40-10) in DI water. These microparticles have a density of $1.27 \mathrm{~g} / \mathrm{cm}^{3}$ and a diameter of 4-5 $\mu \mathrm{m}$ with a smooth surface. Sediment introduces randomness to the process, resulting in different structures. Nonetheless, the three-particle structures can be easily obtained and directly used in our experiments.
During the magnetic actuation, we did not observe deformation of the swimmer by turning on and off the field, which indicates the link between two microparticles is fixed and stable.

Our electromagnetic coils setup consists of three orthogonally placed Helmholtz coil pairs, a swimming tank containing a Si substrate inside and a light microscope with a recording camera on the top. Rotating magnetic field is generated by the coil system (Fig. 8) actuated 
by three servo amplifiers (ADS 50/5 4-Q-DC, Maxon Inc.). The amplifiers are controlled by a LabVIEW program through an Analog and Digital I/O card (Model 826 , Sensoray Inc.), frequency, field strength as well as yaw $(\beta)$ and pitch angles $(\alpha)$ can be adjusted through this program. Schematic of the magnetic field is shown in Fig. 2 b. A swimming tank $(21 \mathrm{~mm} \times 21 \mathrm{~mm} \times 3 \mathrm{~mm})$ filled with DI water is placed in the middle of the coils, and the $\mathrm{Si}$ substrate inside provides a solid surface. The top camera records the motion of the microswimmer at a rate of 50 fps.

\section{Results and discussion}

The microrobot swims away from the solid surface $\left(\alpha=0^{\circ}\right)$ The microswimmer has been actuated at a frequency range from 1 to $16 \mathrm{~Hz}$ on a Si substrate in the tank. The flux density of the magnetic field maintains $9 \mathrm{mT}$ during the experiments. When the input frequency is below $8 \mathrm{~Hz}$, the microswimmer exhibits simple rotation and no translational velocity is observed (Fig. 9a), whereas it exhibits propulsion with varied poses when the input frequency is higher than $8 \mathrm{~Hz}$ (Fig. 9b). Experiment results show that $8 \mathrm{~Hz}$ is the pose-change frequency $\omega_{c}$. When the input frequency is below $\omega_{c}(8 \mathrm{~Hz})$, the microswimmer exhibits simple rotation and the drag torque is small enough to be balanced by the magnetic torque. The projection of rotation axis in $X Y$-plane is closing to the centroid of the simplified triangle gradually with increasing the input frequency, in order to reduce the drag torque (Fig. 4a). Such adjustment of rotation axis cannot affect the actuation pose (simple rotation) of the microswimmer. Equations 14-17 have shown the limitation of this adjustment method, which also explains why the microswimmer cannot maintain simple rotation with input frequency higher than $\omega_{c}$. While when the input frequency is higher than $\omega_{c}(8 \mathrm{~Hz})$, the drag torque is affected by both the pose angle $\varphi$ and the distance $d_{m}$ (Fig. 4c). Different input frequencies of the magnetic field change the drag torque, and dynamic behaviors of the microswimmer are governed by the interplay of magnetic and resistant torques. The dynamic behaviors appear when turning on the magnetic field or changing the input frequency (see Additional file 1). As shown in the experimental results (Fig. 9b), after turning on the magnetic field the dynamic behaviors of the microswimmer last less than $2 \mathrm{~s}(0-2 \mathrm{~s})$. After that the microswimmer exhibits steady rotation and propulsion $(2-29 \mathrm{~s})$.

Swimming velocity of the microswimmer along the $Z$-axis is measured by a fixed distance $\Delta z$ divided by time $\Delta t$. It follows three steps. First, the focal plane of the microscope is set on the substrate, followed by recording and turning the magnetic field on. This step aims to record the starting time. Then, the focal plane is adjusted to $20 \mu \mathrm{m}$ above the substrate. The microswimmer swimming across the focal plane is observed as it became in focus gradually and then out of focus. Finally, we find the best focusing frame from the recorded video to count the time $\Delta t$. Using this method, swimming velocity of the microswimmer in the space 0 to $20 \mu \mathrm{m}$ above the substrate (bottom space) is measured. The velocity in the space 20-40 $\mu \mathrm{m}$ (upper space) above the substrate is measured using the same method. After turning off the magnetic field, the microswimmer will gradually sink onto the substrate due to gravitational force. The
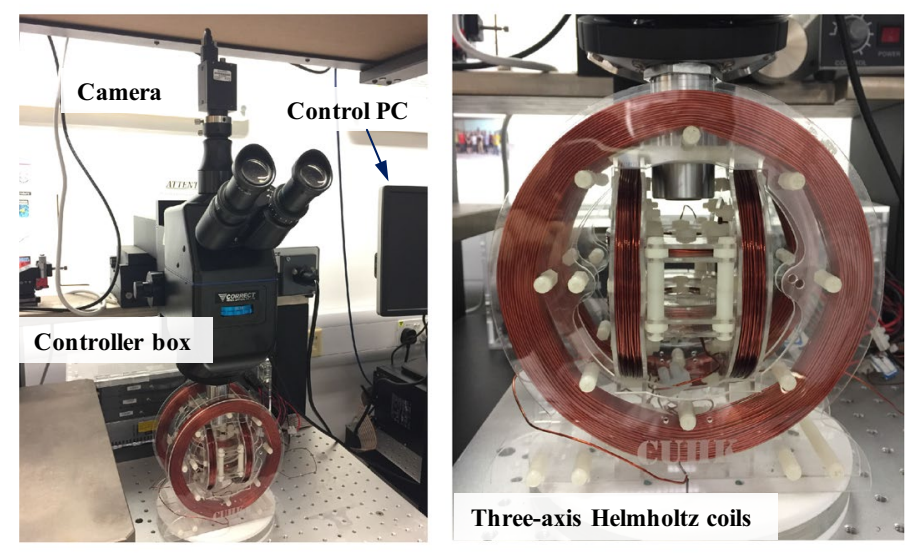

Fig. 8 Magnetic actuation setup. Three-axis Helmholtz electromagnetic coils are applied for generating rotating magnetic field. A camera is mounted on the top of a light microscope for video recording. The actuation setup is controlled by using a PC and a controller box with three amplifiers and one power supply inside 


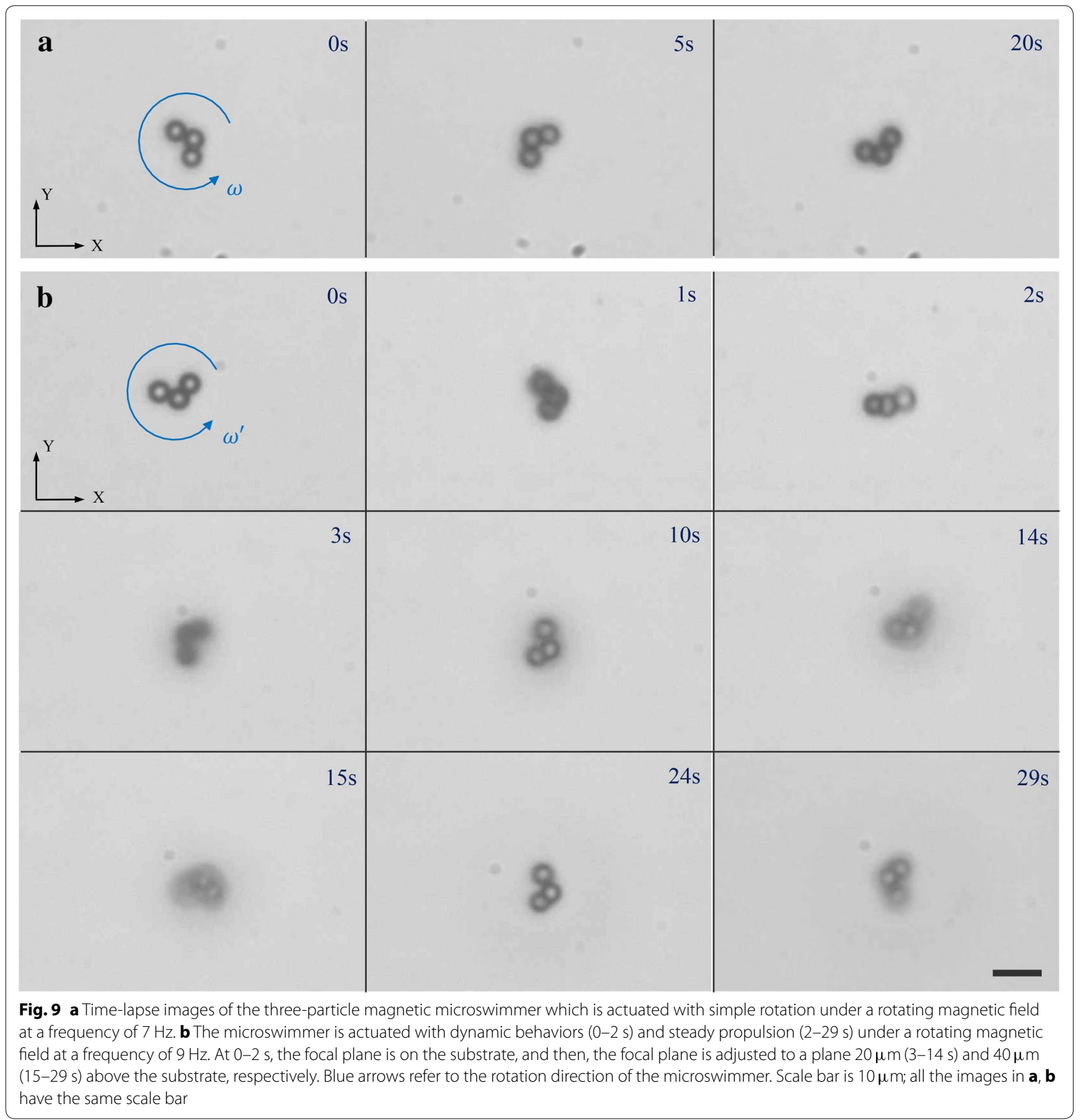

swimming velocity against frequency in the bottom and upper space is depicted in Fig. 10.

Next, we show magnetic steering of the microswimmer. It swims along the direction of $+Z$-axis after exerting field at a frequency of $10 \mathrm{~Hz}$ with $\alpha=0^{\circ}$. After lifting $25 \mu \mathrm{m}$ from the substrate, it can stay in focus by adjusting pitch angle $\gamma$ to $80^{\circ}$, showing negligible displacement along $Z$-direction (see Additiona file 1 ). The propulsive force has the same direction with the normal line of the applied magnetic field. In this scenario, gravitational force and buoyancy are balanced by the component of propulsive force on $Z$-axis. Steering can be done by adjusting the yaw angle $\beta$ of the field from $0^{\circ}$ to $360^{\circ}$ as shown in Fig. 11. The microswimmer did not show visible sidewise drift because of the absence of the boundary effect [33]. The propulsive force is measured based on the equilibrium of forces, which contains gravitational force, buoyancy and propulsive force. The gravitational force 


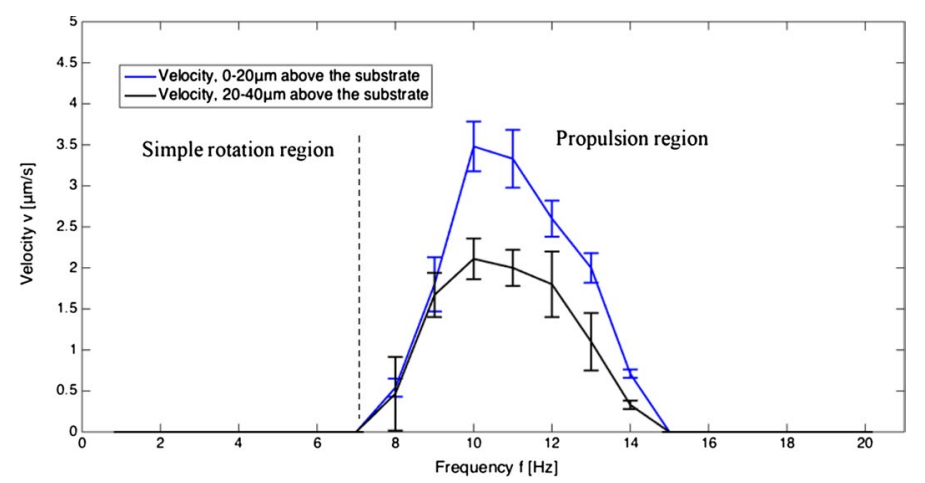

Fig. 10 Velocity of the microswimmer against frequency. Velocity of the microswimmer against frequency of the applied magnetic field. Rotation axis is perpendicular to the solid surface $\left(\alpha=0^{\circ}\right)$. Blue and black lines are the velocity in bottom space $(0-20 \mu \mathrm{m}$ above the substrate) and upper space (20-40 $\mu \mathrm{m}$ above the substrate), respectively. Error bars denote the standard errors from observation time that is used to calculate velocity

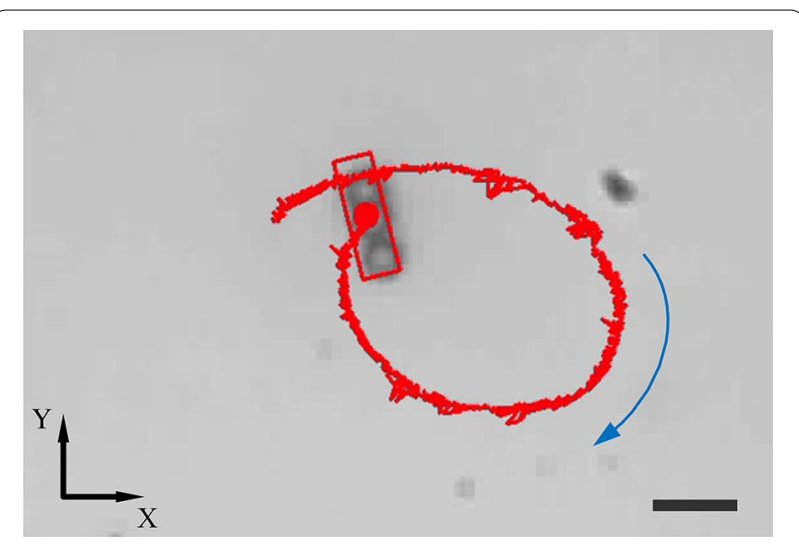

Fig. 11 Steering of the microswimmer. Swimming trajectory (red line) of the microswimmer in a plane $25 \mu \mathrm{m}$ above the substrate (top view). Blue arrow refers to the swimming direction, and red rectangular is applied for tracking the position of the microswimmer. Scale bar is $10 \mu \mathrm{m}$

and buoyancy, respectively, are 1.82 and $1.43 \mathrm{pN}$, and the propulsive force is calculated to be $1.14 \mathrm{pN}$. Based on the simulation results, the net force generated by pressure difference is in the order of piconewton as well, which implies this net force can enhance swimming velocity. Figure 10 shows that the microswimmer has a higher swimming velocity in the bottom space $(0-20 \mu \mathrm{m}$ above the surface), which validates our calculation and simulation results.

\section{Actuation of the microrobot near the solid surface $\left(\alpha=90^{\circ}\right)$}

The microswimmer has been actuated with pitch angle $\alpha=90^{\circ}$ (i.e. its rotation axis is parallel to the horizontal substrate) above the $\mathrm{Si}$ substrate. It shows frequencydependent motion regimes, that is, tumbling (1-3 Hz) and wobbling $(3-20 \mathrm{~Hz})$. The plot of velocity verses frequency is depicted in Fig. 12. When input frequency is below $3 \mathrm{~Hz}$, the microswimmer exhibits tumbling motion with $90^{\circ}$ precession angle. After increasing the input frequency to be higher than $3 \mathrm{~Hz}$, the microswimmer exhibits wobbling motion and the precession angle decreases continually under higher frequency. Previous studies show that dynamic regimes for the tumblingto-wobbling transition of a magnetic microswimmer depend not only on the frequency of the magnetic field, but also on the geometry and easy axis orientation of the microswimmer [41]. In our experiments, when the microswimmer is actuated at a frequency range of $1-3 \mathrm{~Hz}$, both the easy axis and the induced magnetic moment are oriented along the field direction. The easy axis and the induced magnetic moment rotate with a phase lag behind the magnetic field, resulting in tumbling motion of the microswimmer. After increasing the input frequency to be higher than $3 \mathrm{~Hz}$, the drag torque increases and its interplay with the magnetic torque results in wobbling regime. During the experiments, the precession angle of the microswimmer decreases with increasing input frequency of the magnetic field, same as the theoretical prediction [41]. The swimming velocity reaches maximum under magnetic field at a frequency of $10 \mathrm{~Hz}$, similar to the results shown in Fig. 10.

Drifting of the microswimmer occurs due to the boundary effects. The drag coefficient is constant for a certain sphere particle in bulk fluid, but the presence of a solid surface increases the drag on a body, which decreases with a growing distance between the microswimmer and the surface [42]. To be specific, a segment of the microswimmer closer to the surface exhibits larger drag than that farther away the surface, which causes the microswimmer drift sidewise, perpendicular to the rotation axis. Figure 12 also indicates that unlike the $\mathrm{ABF}$ 


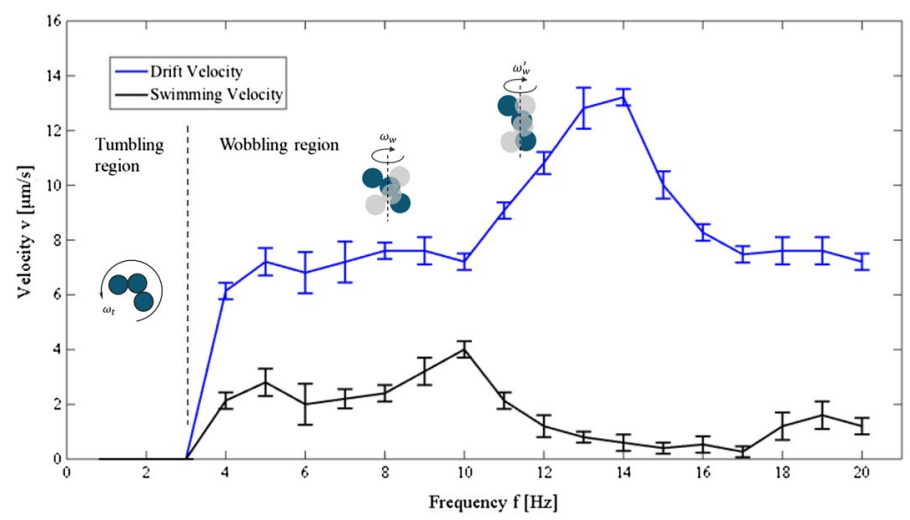

Fig. 12 Velocity of the microswimmer near a solid surface. Swimming and drift velocity of the microswimmer with rotation axis parallel to the solid surface $\left(\alpha=90^{\circ}\right)$. The microswimmer exhibits dynamic behaviors with increasing frequency. The error is from the pixel size of the camera and software Image J

in [33], the drift velocity of the microswimmer is not increasing linearly with the input frequency.

\section{Conclusions}

In this paper, we demonstrate dynamic behaviors of a three-particle paramagnetic microswimmer near a solid surface. These dynamic behaviors are dependent on the input frequency of the rotating magnetic field, and varied actuation poses can be switched by adjusting the frequency. Simulations of the microswimmer near $(0.75 \mu \mathrm{m})$ and far farther away $(20.75 \mu \mathrm{m})$ from a solid surface are investigated, which are in good agreement with the experimental results. Finally, the effects of a solid surface on swimming behaviors are proposed, i.e., enhancing swimming velocity when the microswimmer exhibits propulsion perpendicular to the horizontal surface and causing sidewise drift when it is actuated parallel to the surface. Future studies will focus on the motion control of the microswimmer in biofluids with different viscosities.

\section{Additional file}

Additional file 1. This video demonstrates the microswimmer actuated under a rotating magnetic field at a frequency of $7 \mathrm{~Hz}$ and $9 \mathrm{~Hz}$, and magnetic steering of the microswimmer.

\section{Authors' contributions}

QW designed experiments, built the analytical model and simulation, as well as drafted the manuscript. QW and JY performed the experiments. LY designed the magnetic actuated system. LZ supervised the project and made contributions to the revision of the draft. Part of the work will be presented at the 2017 IEEE International Conference on Robotics and Biomimetics (IEEE ROBIO2017). All authors read and approved the final manuscript

\section{Author details}

${ }^{1}$ Department of Mechanical and Automation Engineering, The Chinese University of Hong Kong, Shatin, Hong Kong SAR, China. ${ }^{2}$ Chow Yuk Ho Technology Centre for Innovative Medicine, The Chinese University of Hong Kong,
Shatin, Hong Kong SAR, China. ${ }^{3}$ Shenzhen Research Institute, The Chinese University of Hong Kong, Shenzhen 518172, China.

\section{Acknowlegements}

The authors thank D. D. Jin (Chinese University of Hong Kong) for the fruitful discussions.

\section{Competing interests}

The authors declare that they have no competing interests.

\section{Funding}

This paper is supported by the Early Career Scheme (ECS) grant with Project No. 439113, the General Research Fund (GRF) with Project No. 14209514, 14203715 and 14218516 from the Research Grants Council (RGC), the ITF project with Project No. ITS/231/15 funded by the HKSAR Innovation and Technology Commission (ITC) and the National Natural Science Funds of China for Young Scholar with Project No. 61305124.

\section{Publisher's Note}

Springer Nature remains neutral with regard to jurisdictional claims in published maps and institutional affiliations.

Received: 9 October 2017 Accepted: 4 November 2017

Published online: 16 November 2017

\section{References}

1. Sitti M, Ceylan H, Hu W, Giltinan J, Turan M, Yim S, Diller E. Biomedical applications of untethered mobile milli/microrobots. Proc IEEE. 2015;103(2):205-24.

2. Nelson BJ, Kaliakatsos IK, Abbott JJ. Microrobots for minimally invasive medicine. Annu Rev Biomed Eng. 2010;12:55-85.

3. Vikram Singh A, Sitti M. Targeted drug delivery and imaging using mobile milli/microrobots: A promising future towards theranostic pharmaceutical design. Curr Pharm Des. 2016;22(11):1418-28.

4. Yan X, Zhou Q, Yu J, Xu T, Deng Y, Tang T, Feng Q, Bian L, Zhang Y, Ferreira $A$, et al. Magnetite nanostructured porous hollow helical microswimmers for targeted delivery. Adv Funct Mater. 2015;25(33):5333-42.

5. Kummer MP, Abbott JJ, Kratochvil BE, Borer R, Sengul A, Nelson BJ. Octomag: an electromagnetic system for 5-dof wireless micromanipulation. IEEE Trans Robot. 2010:26(6):1006-17.

6. Abbott JJ, Peyer KE, Lagomarsino MC, Zhang L, Dong L, Kaliakatsos IK, Nelson BJ. How should microrobots swim? Int J Robot Res. 2009;28(11-12):1434-47. 
7. Peyer KE, Tottori S, Qiu F, Zhang L, Nelson BJ. Magnetic helical micromachines. Chem-A Eur J. 2013;19(1):28-38.

8. Peyer KE, Zhang L, Nelson BJ. Bio-inspired magnetic swimming microrobots for biomedical applications. Nanoscale. 2013;5(4):1259-72.

9. Yu J, Xu T, Lu Z, Vong Cl, Zhang L. On-demand disassembly of paramagnetic nanoparticle chains for microrobotic cargo delivery. IEEE Trans Robot. 2017:33(5). https://doi.org/10.1109/TRO.2017.2693999.

10. Yang L, Wang Q, Vong C-I, Zhang L. A miniature flexible-link magnetic swimming robot with two vibration modes: design, modeling and characterization. IEEE Robot Autom Lett. 2017;2(4):2024-31.

11. Zhang L, Abbott JJ, Dong L, Kratochvil BE, Bell D, Nelson BJ. Artificial bacterial flagella: fabrication and magnetic control. Appl Phys Lett. 2009;94(6):064107.

12. Zhang L, Abbott JJ, Dong L, Peyer KE, Kratochvil BE, Zhang H, Bergeles $C$, Nelson BJ. Characterizing the swimming properties of artificial bacterial flagella. Nano Lett. 2009;9(10):3663-7.

13. Tottori S, Zhang L, Qiu F, Krawczyk KK, Franco-Obregón A, Nelson BJ. Magnetic helical micromachines: fabrication, controlled swimming, and cargo transport. Adv Mater. 2012;24(6):811-6.

14. Tottori S, Zhang L, Peyer KE, Nelson BJ. Assembly, disassembly, and anomalous propulsion of microscopic helices. Nano Lett. 2013;13(9):4263-8.

15. Zhang L, Peyer KE, Nelson BJ. Artificial bacterial flagella for micromanipulation. Lab Chip. 2010;10(17):2203-15.

16. Ghosh A, Fischer P. Controlled propulsion of artificial magnetic nanostructured propellers. Nano Lett. 2009;9(6):2243-5.

17. Fischer P, Ghosh A. Magnetically actuated propulsion at low Reynolds numbers: towards nanoscale control. Nanoscale. 2011;3(2):557-63.

18. Maier AM, Weig C, Oswald P, Frey E, Fischer P, Liedl T. Magnetic propulsion of microswimmers with DNA-based flagellar bundles. Nano Lett. 2016;16(2):906-10.

19. Helgesen G, Pieranski P, Skjeltorp AT. Nonlinear phenomena in systems of magnetic holes. Phys Rev Lett. 1990;64(12):1425.

20. Helgesen G, Pieranski P, Skjeltorp A. Dynamic behavior of simple magnetic hole systems. Phys Rev A. 1990;42(12):7271.

21. Sandre O, Browaeys J, Perzynski R, Bacri J-C, Cabuil V, Rosensweig R. Assembly of microscopic highly magnetic droplets: magnetic alignment versus viscous drag. Phys Rev E. 1999;59(2):1736.

22. Gao W, Feng X, Pei A, Kane CR, Tam R, Hennessy C, Wang J. Bioinspired helical microswimmers based on vascular plants. Nano Lett. 2013;14(1):305-10.

23. Mahoney AW, Nelson ND, Peyer KE, Nelson BJ, Abbott JJ. Behavior of rotating magnetic microrobots above the step-out frequency with application to control of multi-microrobot systems. Appl Phys Lett. 2014;104(14):144101.

24. Ghosh A, Paria D, Singh HJ, Venugopalan PL, Ghosh A. Dynamical configurations and bistability of helical nanostructures under external torque. Phys Rev E. 2012;86(3):031401.
25. Vach PJ, Brun N, Bennet M, Bertinetti L, Widdrat M, Baumgartner J, Klumpp S, Fratzl P, Faivre D. Selecting for function: solution synthesis of magnetic nanopropellers. Nano Lett. 2013;13(11):5373-8.

26. Vach PJ, Fratzl P, Klumpp S, Faivre D. Fast magnetic micropropellers with random shapes. Nano Lett. 2015;15(10):7064-70.

27. Kei Cheang U, Lee K, Julius AA, Kim MJ. Multiple-robot drug delivery strategy through coordinated teams of microswimmers. Appl Phys Lett. 2014;105(8):083705.

28. Cheang UK, Meshkati F, Kim D, Kim MJ, Fu HC. Minimal geometric requirements for micropropulsion via magnetic rotation. Phys Rev E. 2014:90(3):033007.

29. Morozov KI, Mirzae Y, Kenneth O, Leshansky AM. Dynamics of arbitrary shaped propellers driven by a rotating magnetic field. Phys Rev Fluids. 2017;2(4):044202

30. Lauga E, DiLuzio WR, Whitesides GM, Stone HA. Swimming in circles: motion of bacteria near solid boundaries. Biophys J. 2006;90(2):400-12.

31. Lauga E, Powers TR. The hydrodynamics of swimming microorganisms. Rep Prog Phys. 2009;72(9):096601.

32. Riedel IH, Kruse K, Howard J. A self-organized vortex array of hydrodynamically entrained sperm cells. Science. 2005:309(5732):300-3.

33. Peyer KE, Zhang L, Kratochvil BE, Nelson BJ. Non-ideal swimming of artificial bacterial flagella near a surface. In: Robotics and Automation (ICRA) 2010 IEEE International Conference on. 2010, pp. 96-101.

34. Ledesma-Aguilar R, Yeomans JM. Enhanced motility of a microswimmer in rigid and elastic confinement. Phys Rev Lett. 2013;111(13):138101.

35. Zhou Q, Petit T, Choi H, Nelson BJ, Zhang L. Dumbbell fluidic tweezers for dynamical trapping and selective transport of microobjects. Adv Funct Mater. 2017;27(1). https://doi.org/10.1002/adfm.201604571.

36. Happel J, Brenner H. Low Reynolds number hydrodynamics: with special applications to particulate media, vol. 1. Berlin: Springer; 1983.

37. Biswal SL, Gast AP. Rotational dynamics of semiflexible paramagnetic particle chains. Phys Rev E. 2004;69(4):041406.

38. Doi M, Edwards SF. The theory of polymer dynamics, vol. 73. Clarendon: Oxford University Press; 1988.

39. Liu B, Breuer KS, Powers TR. Propulsion by a helical flagellum in a capillary tube. Phys Fluids. 2014;26(1):011701.

40. Wu G. Fluid impact on a solid boundary. J Fluids Struct. 2007:23(5):755-65.

41. Morozov KI, Leshansky AM. Dynamics and polarization of superparamagnetic chiral nanomotors in a rotating magnetic field. Nanoscale. 2014;6(20):12142-50.

42. Brennen C, Winet H. Fluid mechanics of propulsion by cilia and flagella. Annu Rev Fluid Mech. 1977;9(1):339-98.

\section{Submit your manuscript to a SpringerOpen ${ }^{\circ}$ journal and benefit from:}

- Convenient online submission

- Rigorous peer review

- Open access: articles freely available online

- High visibility within the field

- Retaining the copyright to your article

Submit your next manuscript at $\boldsymbol{\nabla}$ springeropen.com 